\title{
Local Satisfaction explained away
}

\author{
Henk Zeevat \\ ILLC, University of Amsterdam \& \\ SFB991, Heinrich-Heine-Universität \\ Düsseldorf
}

\begin{abstract}
The paper makes the proposal to replace local satisfaction as a source of presupposition blocking by causal and identity inferences that indirectly - through Trapping - lead to the same effect in simplest cases and to much better predictions in the more complex cases.
\end{abstract}

Keywords: presupposition projection, local satisfaction, causal inferences, identity inferences, trapping, consistency, epithets, interjections

\section{The Local Satisfaction Exception}

Underlying the satisfaction theory of presupposition is the local satisfaction exception (LSE), an empirical generalisation that can be stated as in (2). ${ }^{1}$ The LSE explains why in (1a), the presupposition John killed Mary triggered by the presupposition trigger regret is not projected, i.e. inferred to be a speaker assumption, while in cases where the antecedent does not entail the presupposition as in (1b), it is.

(1) a. If John killed Mary, he regrets killing her.

b. If John loved Mary, he regrets killing her.

(2) LSE:

If $B$ presupposes $P$ in a context $C$ and $C+A \models P$ then $A \rightarrow B$ does not presuppose $P$ in $C$.

While logical entailment $(=)$ gets its observational content by semantic intuitions of entailment and it should be transparent what is in the context $C$ and $\rightarrow$ can be fixed to the English if ... then-construction, the relation presuppose does not have an uncontroversial observational content.

1 LSE derives from Karttunen (1974) though Karttunen does not accept the Cumulative Hypothesis in that paper (or later), so that it is not properly an exception in that paper. It becomes an exception with Gazdar (1979) and Van der Sandt (1988) and the authors that mix Gazdar with Karttunen such as Soames (1982); Heim (1983); Van der Sandt (1992); Zeevat (1992). 
In this paper, the choice is made for (3) as that observational content, mainly because other formulations have even more problems. The choice is different from making presupposition a categorical property of sentences. Presuppositions are merely a special category of inferences, attendant on the use of presupposition triggers in utterances.

(3) $B$ presupposes $P$ iff a felicitous use of $B$ normally leads to the inference of the hearer that the speaker assumes that $P$ and $P$ can be connected to the occurrence of a presupposition trigger in $B$ associated with the presupposition $P$

The weak spot in this formulation is normally. It requires that one should be able to spot the special property of the context that prevents the inference when it does not occur and that by removing the special property the inference is restored. The second clause requires that the inference disappears if the trigger is replaced by a neutral construction or lexical item.

The stipulation of the empirical content of $B$ presupposes $P$ is needed to make sense of the cumulative hypothesis of Langendoen \& Savin (1971), the empirical generalisation behind the battery-of-tests diagnosis of presupposition.

\section{(4) Cumulative Hypothesis}

A sentence $B$ with a presupposition trigger for $P$ presupposes $P$

The LSE gives a straightforward exception to the cumulative hypothesis.

Given these stipulations, LSE has enough empirical content to note that it is correct in the obvious cases. It moreover makes philosophical sense on the view going back to Frege that presuppositions are conditions on the context for the use of certain expressions, in Frege's case the definite description. The fact that $A \models P$ makes it the case that $B$ can be used in $A \rightarrow B$ and that $A \rightarrow B$ does not make the truth of $P$ a condition on the context.

LSE is assumed in all known accounts of presupposition projection ${ }^{2}$, from the plugs, holes and filters account of Karttunen (1973), to the satisfaction account of Karttunen (1974) and Karttunen \& Peters (1979), the accounts of Heim (1983), of Gazdar (1979) and Van der Sandt (1988), Soames (1982), Van der Sandt (1992), Zeevat (1992) and Rothschild (2011) and the accounts of Schlenker (2007) and those following up on these. Crucially, the accounts of Gazdar (1979) and Van der Sandt (1988) that seek a different way of capturing the effects of LSE have been shown to fail on the cases where $A \models P$ but $P \not \models A$. Notice that with the exception of

2 With the exception of Garcia-Odon (2016) whose argument that LSE could never work for proper causal examples like If Mary is French, she has stopped eating snails was the inspiration for the work reported in this paper, but whose alternative explanation based on conditional perfection is different. 
Van der Sandt (1992) which uses a version of strong LSE based on formal identity, all these accounts capture a stronger version of LSE, which generalises LSE from the particular case of the consequent of an implication to any local context as in (5).

(5) $\quad$ strong LSE: If $B$ presupposes $P, C \models P$ and $B$ occurs in $A$ in a position where $C$ is assumed then $A$ does not presuppose $P$

While strong LSE seems to be about as plausible as LSE from a philosophical perspective, it does not qualify as an empirical generalisation, since the question what is locally assumed is theoretical and cannot be given empirical content in a direct way. If LSE were correct, strong LSE would be the correct generalisation to capture in accounts of presupposition, especially since — unlike LSE — it would seem to capture precisely the limitations of the Cumulative Hypothesis that do not follow from general constraints on interpretation such as consistency and the absence of free variables.

This paper however argues that LSE is both too strong and too weak in an empirical sense and proposes a different but related empirical generalisation to take its place.

\section{Causal and Identity Inferences}

Consider (6). For (6a), everybody infers that John fell because Bill pushed him, for (6b) nobody infers that John fell because Mary smiled at him, while (6c) shows that the inference for (6a) is not necessary and (6d) shows that it is perfectly consistent to fall because Mary smiles at you (you must be a bit shy presumably). (6a) is an example of a causal inference. The hearer sees a causal connection that is not overtly marked and the speaker intends the hearer to see it.

(6) a. John fell. Bill pushed him.

b. John fell. Mary smiled at him.

c. John fell. Then Bill pushed him.

d. John fell. Because Mary smiled at him.

For (7a) (from Jasinskaja 2007), everybody infers that the breaking of the skis is the loss of the only means of transport and also that the skis are the only means of transport. Without any overt marking, the hearer sees the two identities and this is as intended by the speaker. One can mark against making these identity inferences. For example, an additive particle also on lost as in (7b) removes the inference.

(7) a. Alena broke her skis. She lost her only means of transport.

b. Alena broke her skis. She also lost her only means of transport. 
Local Satisfaction explained away

Causal and identity inferences happen all the time in understanding natural language utterances and in understanding visual information. In (8), hearers identify she with the wife of the first sentence.

(8) John has a wife. She is happy.

In (9), one infers that the event in the first sentence caused John to have a wife.

(9) John got married. His wife is happy.

Just as in (10), one infers a causal connection between John's being a diver and his possession of a wetsuit.

(10) John is a diver. He has a wetsuit.

The same type of inferences abound in interpreting visual information. If I see a jogger disappearing behind a bus and emerge some seconds later on the other side, I have identified my two jogger appearances. If I see John running in the direction of the bus stop and a bus approaching at the street corner, I infer that John is running in order to catch the bus. i.e. that John's desire to catch the bus causes him to run towards the bus stop. These inferences are the bread and butter of visual perception and of NL understanding. ${ }^{3}$

While causal and identity inferences clearly belong to NL pragmatics, Gricean pragmatics seems to omit them. They have received attention in discourse semantics and especially in the study of discourse relations (Hobbs 1985; Asher \& Lascarides 2003; Jasinskaja 2007; Kehler 2002; Kehler, Kertz, Rohde \& Elman 2008), but, even there, there is no systematic treatment.

\section{Causal and Identity Inferences and presupposition blocking}

(11) is the empirical generalisation that should replace the LSE.

(11) CIG(causal and identity inference generalisation):

Whenever one encounters an LSE-type exception to the cumulative hypothesis, then there is a causal or identity inference in the text "A.P." where A is the antecedent of the conditional and $\mathrm{P}$ the presupposition of the trigger in the consequent.

3 It seems reasonable to connect these inferences with the categories of substance and causality in the philosophy of Kant. That makes them basic for our conception of reality as consisting of objects independent of our perceptions that go through changes that are also independent of our perceptions. 
There being a causal or identity inference will be a question of semantic intuition in these pages. The author is working on an paper which outlines a predictive theory of these inferences, but where - as it should be - an important role is given to subjects learning stochastic causal generalisations from experience. Given that there is no extant machine-readable result of the extensive learning processes required that one could slot into the theory, it is to ourselves - as the closest approximations to such results - that one must turn for judgments on these inferences. Notice that these judgments are far less problematic than e.g. grammaticality judgments where there is no generally accepted account that explains where they come from.

The empirical claim can explain presupposition blocking as follows. Assume that both causal and identity inferences bind a variable in the representation of the content of the event or state that is caused by or identified with a given object. In implications, that variable is then bound from the antecedent of the conditional by a discourse referent that is - seen from the outside - merely an object that might exist. Projection of the presupposition with the bound variable to the common ground would then result in the variable become unbound, i.e. in the full interpretation becoming meaningless by containing free variables.

On this account, predicates of events (and certain classes of predicates of spatiotemporal continuants) give rise to reports of events in which they have certain given causes or are constituting or being constituted by certain given events. These causes or constituting other events are not necessarily present: if no cause, constituter or constituent is given, i.e. no causal or identity inferences are made, the report is of an event that is not caused by any specified cause and is distinct from any given event.

What blocks projection therefore in these cases is exactly the same as what prevents the projection of he ${ }_{i}$ has a mother in (12).

\section{(12) Every boy likes his $_{i}$ mother}

It will not project because outside the scope of every boy $y_{i}, h e_{i}$ is meaningless. The general principle has been called Trapping, and goes back to Van der Sandt (1992). The Trapping principle has nothing to do with presupposition as such, it is merely a condition on what is a meaningful logical representation. For example, it also prevents the wide scope reading of his $s_{i}$ mother in (12).

In this respect, it is like the other widely accepted constraint on presupposition projection, Consistency. This is also a general constraint on interpretations and vital for phonological, morphological, lexical and syntactic disambiguisation and for pronoun resolution and recognition of speech acts and discourse structure. An inconsistent interpretation just is not useful for inference. ${ }^{4}$ Later on in this paper, it

4 Corrections are the exception in that they can be inconsistent with the current context. However they should not be seen as statements inconsistent with the context, but as instructions to change that 
will be shown that Trapping and Consistency are also relevant for projection from epithets, interjections and non-restrictive modifiers.

Unlike what is the case for LSE, there is a general observational principle related to CIG above that can be formulated as in (13).

(13) If the presupposition is inferred to be caused by or identical with a referent that is given by a non-entailed clause in the sentence, it does not project.

Non-entailed clauses can be easily identified, following the pioneering work of Karttunen (1973).

In the rest of this section, the LSE will be compared with the CIG.

Same predictions In many cases, the predictions from LSE are identical with those resulting from the alternative approach introduced here, in fact all the evidence for LSE is evidence for the CIG. (14) would be the standard example, though $P$ 's identity with $A$ makes $A$.P. pragmatically unacceptable, when an I repeat or a similar device is not employed.

(14) If John has a wife, his wife is happy.

John has a wife. John has a wife. (identity inference)

(15) is closely related and explained by a causal inference under the CIG and — this will be challenged later on- by entailment under the LSE

(15) If John got married, his wife is happy.

John got married. He has a wife. (causal inference)

It is hard to make an empirical case for LSE against Van der Sandt (1992)'s moderate version of LSE in which one demands formal identity instead of logical entailment. The best counterexamples are indeed cases like (15) where world knowledge and conceptual knowledge needs to be invoked for weak LSE so that LSE seems to have the advantage over Van der Sandt's version at this point.

Formal identity, logical entailment and strong probability Identity inferences however do not require formal identity or logical entailment (LSE) or strong probability. (16a,b) are clear cases. The identity is inferred for the sake of coherence between $A$ and $P$ and the inference can easily be prevented by additive particles. It is not probable that people driving into the canal are 44-year old plumbers or that skis are one's only means of transport.

context. 
(16) a. A man drove into the canal. The 44-year old plumber managed to escape from the car.

b. Alena broke her skis. She lost her only means of transport.

(16b) leads to presupposition blocking as in (17).

(17) If Alena broke her skis, she is unhappy that she lost her only means of transport.

From (16a) one obtains the deviant (18). This is presumably because one cannot explain how the speaker could know that an arbitrary person driving into the canal would be a 44-year old plumber. In contrast, speakers could know that Alena's skis are her only means of transport.

(18) ?? If someone drove into the canal, Mary is happy that the 44-year old plumber escaped.

Causal inferences The causal inferences lead to the same complex situation. They never involve formal identity and the situation where they lead to logical entailment given extra world knowledge is not common, if it occurs at all. ${ }^{5}$

In fact, there is no logical entailment from John got married to John has a wife, not even accepting John is male and operating under a legal system where men can only marry women. John might have obtained a divorce after getting married, or his spouse could have died. Causality in general does not seem to be reducible to logical entailment given that ceteris paribus always needs to be assumed. Causal inferences also do not seem to need a particularly strong causal connection, it suffices that the probability of the effect goes up significantly compared to its probability without the cause. As an example consider the variant (19) of (16) .

(19) If John got married, his husband is happy.

John got married. He has a husband. (causal inference)

While the probability of having a same sex spouse rather than a different sex spouse if one has got married is rather small, it is very much higher than if one did not get married at all.

5 The best examples where causal knowledge can lead to conceptual entailment are from the legal domain. It can be world knowledge that John owns a house and signs a deed making Mary the owner. Then from John signed the deed it follows that Mary owns John's house. And If John signed the deed, Mary owns John's house is indeed a case of presupposition blocking. But even here there can be extra formalities required by law (e.g. witnesses watching the signature, or John being in full possession of his mental capacities). It is therefore not even here merely a question of concepts. 
Karttunen started the discussion of these cases with his holy underwear example in (Karttunen 1974). Much the same holds for his example as for the examples in (20).

(20) a. If Mary is French, she has stopped eating snails.

Mary is French. She eats snails.

b. If John is a diver, he will bring his wetsuit to the party.

John is a diver. He has a wetsuit.

c. If John is a diver, he will show you his titanium mouthpiece.

John is a diver. He has a titanium mouthpiece.

The important point is that while there are clear causal inferences in these cases (the c-example is fictional: a titanium mouthpiece would be a useful but expensive piece of diving gear that only the richest divers can afford to buy), the causal inference is far from a logical entailment. There is a causal connection in (20a). In France, people do eat snails and most French people will be offered snails at some point in their lives and try them, and, since many people like snails when they try them, join in with the habit of eating snails. But while many French people enjoy their snails, there are also many French vegetarians and many French people who just do not like to eat snails. And this is general knowledge. It is thereby not possible to make Mary's eating snails a logical consequence of world knowledge and the fact that Mary is French. The non-projection of the presupposition in (20a) therefore cannot be explained by the LSE. The probability of eating snails if one is French is however much higher than for British or Dutch people. Similarly, divers will have wetsuits, but not every diver will. It is not a question of logic and world knowledge that one has a wetsuit if one is a diver, though in this case, any given diver will probably have one. (20c) shows however that the causal inference and the resulting presupposition blocking does not require a high probability, but merely an important increase of $P$ 's probability if $A$ is its cause with respect to $P$ 's probability when $A$ is not its cause.

In sum, contrary to what Karttunen suggests in (Karttunen 1974), LSE cannot be extended to this group of examples by allowing extra premisses from world knowledge. It also does not do to extend logical entailment to "make the conclusion very probable". (20c) and (19) are counterexamples to that view.

(20c) can be turned around to be (21), where the presupposition clearly projects.

(21) If John owns a titanium mouthpiece, he is happy to be a diver.

While it is reasonable to assume that some $3 \%$ of divers own a titanium mouthpiece, almost no non-divers will own one, due to its price and its uselessness for this group of the population. That makes it nearly certain that someone with a titanium 
mouthpiece is a diver. Yet, this is not enough for preventing projection, while the $3 \%$ is enough to block it in the other direction. This can be taken as conclusive evidence against a probabilistic repair of LSE where showing that the presupposition is nearly certain given the antecedent would be enough to give blocking.

Causal inversion The following (22) is an old counterexample to LSE, due to Van der Sandt (1988).

(22) If John has grandchildren, his children are happy.

Most people prefer the reading in which John has children (i.e. the presupposition is projected) over the more marginal reading (which not everybody finds acceptable) in which it is also an open issue whether John has children. ${ }^{6}$

On the current account, the preference is easily explained. Children cause one to have grandchildren and grandchildren do not cause one to have children. Since one cannot have grandchildren without having children, LSE predicts that the projecting reading is completely out, thus coming in conflict with most people's intuitions.

Examples of this kind require unique and imperfect causes. Assume the bomb and the button are so wired up that the bomb cannot explode unless the button is pressed, but only if it is pressed really hard. (24) projects even while presupposition $P$ is entailed under the circumstances by the antecedent $A$.

(24) If the bomb exploded, it wasn't John who pressed the button.

Projected: someone pressed the button. (no causal inference from A to P. )

(25) however does not project, even though pressing the button is not a sufficient condition for the bomb exploding.

(25) If John pressed the button, he is glad the bomb exploded. John pressed the button. The bomb exploded. (causal inference)

Beyond implications As noted above, CIG easily generalises as an empirical generalisation. The antecedent of a conditional is merely one example of a nonentailed clause in a larger sentence. If causal and identity inferences happen from such clauses to presuppositions, they will stop projection in the same way.

And in fact they do. (26a-c) are variations of McCawley's LBJ examples. (26a) is the projecting control (unless one makes the alcoholism causally responsible for

6 The author thinks that the sequence in (23) is acceptable, but there are people who disagree:

(23) \% I do not know whether John has children. If he has grandchildren, his children are happy.

This would be a case in which Consistency prevents projection. 
the failure of the foreign policy), in (26b) and (26c) projection is blocked due to causal and identity inferences respectively.

(26) a. LBJ dreamt that he was an alcoholic and that everybody knew his foreign policy was a failure.

projection: LBJ's foreign policy was a failure. (no obvious causal inference)

b. LBJ dreamt that he was an alcoholic and that everybody knew he was hiding bottles in the Oval Office.

LBJ is an alcoholic. He is hiding bottles in the Oval Office. (causal inference)

c. LBJ dreamt that he was an alcoholic and that everybody knew he was drinking two bottles of whiskey every day.

LBJ is a alcoholic. He is drinking two bottles of whiskey every day. (identity inference)

Empirical conclusions The conclusion that this section and the last one argued for is that the correct observations on which the LSE is based do not warrant the LSE, but only the weaker version of Van der Sandt (1992). Both the weak version and LSE however do not capture the full class of exceptions because they cannot handle any of the cases that involve uncertain inference. The cases which Karttunen (1974) claims that can be handled by using extra world knowledge do not fall under the LSE, since the required extra knowledge typically does not exclude exceptions and so never leads to logical entailment. Extending LSE to include probabilistic reasoning does not help, since it does not remove the counterexamples to the bare LSE while creating many new counterexamples. Moreover, LSE does not give rise to a genuine observational generalisation because it relies on the theoretical notion of "local context".

The alternative sketched where absence of projection is explained by causal and identity inferences leading to Trapping generalises the weak version of LSE to other non-entailed clauses of larger sentences. It seems the correct generalisation from these data. Often one can promote or inhibit the inferences by manipulating the linguistic form or the context. The resulting presence and absence of projection gives powerful support to CIG.

Some words of clarification of the empirical generalisation are in order. Identity inferences are often just that, but in many cases constituency seems the better term. John saying something nasty to Mary constitutes an insult of Mary by John, but it is not the same event, at least for most authors on this matter. The same words could have been said without this being an insult. In other cases, partial identity or partial constituency is the right notion.

The same holds for causality. In this context, one is committed to the Humean view that the judgment that $A$ causes $B$ is the judgment that, without $A, B$ would 
not have happened. But there can be many such $A$ s for a given $B$, summing up to a complex maximal cause, without any of the $A$ s thereby losing the property of being a cause of $B$. There is no alternative to Hume here if one wants to use cause as whatever underlies causal connectives in natural languages, covering physical causation, various factors that shape the will of agents, causes as in reasoning over dynamic models as in the social sciences or in evolutionary causation, or biological causation as in procreation. The underlying mechanisms can be quite diverse, but what these different causal judgments share is that they come with a belief that the effect would not have been there without the cause. The relevant notion for the purposes of this paper is as diverse as what is needed for the analysis of causal connectives and the counterfactual theory is neutral between complete and partial causes.

Identity inferences come with similar counterfactuals. Without John, there would not be his arm. Without John saying those words, the insult to Mary would not be there. The difference is the temporal order in causation. The link with counterfactuals forms a connection between causal and identity inferences.

Eliminating local satisfaction in favour of causal and identity inferences may however seem a desperate move. The theoretical understanding of local satisfaction leaves little to be desired, causal and identity inferences are not very well understood, even though they seem central to both the interpretation of visual signals and natural language. In the last case, anybody who has worked on such topics as discourse coherence, anaphora, tense and aspect or discourse particles will readily admit the central importance of these inferences. It is however not a question of obscurus per obscurior. Accounts that start from empirical LSE (really all approaches) are prone to counterexamples (with the exception of mild LSE as in Van der Sandt (1992)) and fail to work where logic stops. In short, they do not qualify as accounts of presupposition projection. And there is nothing surprising that a discourse phenomenon like presupposition projection needs to be accounted for with the same causal and identity relations that one needs for other discourse phenomena.

This concludes the non-speculative part of the paper. The next two sections outline a more comprehensive account of presupposition and give a first outline of an account of causal and identity inferences.

\section{Consequences for a theory of presupposition}

In recent work (Potts (2005); Simons, Tonhauser, Beaver \& Roberts (2011)), it has been pointed out that presupposition is not the only source of projection. In particular, also epithets, non-restrictive modifiers and interjections give rise to projection, i.e. to inferences that the speaker has the attitude associated with the epithet or accepts the truth of the modifier or the interjection. 
The explanation of these additional kinds of projection seems straightforward. Non-restrictive modifications can be seen as syntactically integrated extra assertions about the referent of the modified item, extra assertions that are on the same level as the main clause which does not need to be an assertion itself. Even more obviously interjections can be seen as additional assertions that are not syntactically integrated and are mostly but not necessarily relevant to the utterance in which they appear. Epithets are lexical items that express particular attitudes to their referents.

Now it seems by far the simplest explanation of the use of an epithet that the speaker has the attitude to the referent and wants to express it. The same holds for the extra assertions. The simplest explanation of the use of a non-restrictive modifier or interjection is that the speaker believes its content and thinks the extra information is relevant to the utterance in which it appears. This extends to the use of presupposition triggers, if one accepts with Frege that these are expressions that put restrictions - expressible as the truth of certain propositions - on the context in which they are used. By far the simplest explanation of their use is that the speaker believes in the truth of these propositions.

This is enough to motivate cumulative principles in all four cases. It is important to notice that there are also exceptions in all cases. The simplest exceptions are the ones where projection breaks the consistency of the speech act or the content of the utterance. In (27), sincerely uttered, the speaker denies that he is is dishonest and cannot be taken as morally disapproving of himself for that reason.

\section{I am not a crook.}

(28) projects. The speaker commits to the anger of the farmers.

(28) The - angry - farmers blocked the road.

But in (29), we get a causal inference, from the lowering of the milk price to the anger of the farmers, that makes projection impossible.

(29) Whenever the milk price is lowered, the farmers - they get very angryblock the roads.

A simple example of Trapping is (30).

(30) If we invite a professor — a famous one - we organise a drink.

So, it seems one can argue for one single cumulative hypothesis and two exceptions to that: Consistency and Trapping, while maintaining the extrapolation of Frege's view on definite descriptions as the theory of presupposition triggers: lexical 
items and constructions that require the truth of certain propositions in their context of use.

There are however three issues that need to be raised.

Consistency versus low plausibility Normally, the best explanation of a trigger is a belief of the speaker. But not invariably. The explanation of the trigger needs to be balanced with everything else that needs to be explained about the utterance in the context. That means that other factors can come in too. Inconsistency is merely a dramatic way in which projection can be a stochastic loser.

The subtle facts studied in Beaver (2010) can be brought under this heading. In (31), it can be practically ruled out that the speaker knows anything about the matter, i.e. the speaker's belief in the presupposition is highly unlikely, even if logic allows the speaker to know that she was cheating.

(31) Did you realise at any point that she was cheating on you?

The same point can be argued from a variant (33) of Beaver's example (32) which works by Consistency.

(32) If Spaceman Spiff lands on planet X, he will be surprised he weighs more than what he weighs on Earth.

The inconsistency of weighing more than what you weigh on Earth can be matched by weighing $300 \mathrm{~kg}$, the outer limit of human weights on earth (extreme obesity), making it unlikely that one will be recruited as a spaceman (and also making it unlikely that one would be surprised by having such a weight). Like in (32), one seems to infer in (33) that the $300 \mathrm{~kg}$ is what Spiff weighs if he lands on planet X.

(33) If Spaceman Spiff lands on planet X, he will be surprised he weighs $300 \mathrm{~kg}$.

This points in the direction of integrating projection into a general account of interpretation in which interpretation is a stochastically-based choice between a number of complete candidate interpretations. This is exactly what the next section proposes for causal and identity inferences and integrating projection in that set-up too makes good sense. The cumulative hypothesis in such a set-up changes into a generalisation on likelihood probabilities. The relevant belief/attitude of the speaker should make the choice for the trigger/epithet/interjection/non-restrictive modifier much more likely than the absence of such a belief with an alternative explanation.

Notice that Trapping has a different status. It is a constraint that rules out candidate interpretations as not semantically well-formed. Consistency follows from 
choosing stochastic maxima, but by itself it is also a good candidate for being a constraint on possible interpretations: inconsistent interpretations are useless for inference.

The following counterexample to CIG (34) is due to a reviewer of the SALT abstract on which this paper is based.

(34) If John has an illegitimate child, he hid his extramarital affair really well.

The example is interesting because it indeed falls under the LSE if sufficient information is added to the context (the birth date of the putative illegitimate child, John's marriage and its date, etc.). It however also allows an explanation in terms of Consistency or Low Plausibility. Notice that it is unlikely the speaker would speak of John's extramarital affair as being well-hidden if he knew about it. So projection may well lose from non-projection. In the variant (35) projection is clearly preferred, even if all the extra facts for (34) are added to the context, in contradiction to the LSE.

(35) If John has an illegitimate child, something resulted from his extramarital affair.

Accommodation Many presupposition triggers do not allow proper accommodation, as has been observed for pronouns (Kamp \& Reyle 1993), "short" descriptions and short names, additive and other particles (Kripke 2009; Zeevat 2002) and intonationally marked givenness. This can be explained from their discourse function in the case of pronouns and particles and in the case of intonationally marked givenness by the nature of the marking device (material to be accommodated is new and needs more stress to ensure it is properly understood). These can be distinguished from the other triggers by requiring that the presupposition is not some proposition $p$ but the proposition that $p$ is common ground, as proposed in (Beaver \& Zeevat 2007). Since hearers know what is common ground, assuming that $p$ is common ground when it is not is inconsistent.

Neither the LSE nor the CIG needs to rule out accommodations for these cases. But this class of triggers is essential for identity inferences, both in forcing them to be made and in preventing them.

Local and intermediate accommodation Under Trapping and Consistency (and in cases where projection is not preferred), projection will not occur, but the utterances will be acceptable which means that by some means or other the proposition required by the trigger is available to the trigger — as predicted by the Fregean take on triggers adopted in this paper-, while the simplest way of bringing this about, assuming that it is a speaker belief, is not available. 
Obviously, the interpreter can adopt alternative explanations of the use of the trigger, e.g. that the speaker is adopting the perspective of somebody else or is speaking under a special assumption, etc. These should be regarded as marked options, but they often give the effect of local and intermediate accommodations. (36) illustrates this for the case of epithets, where the extra assumption is that the speaker thinks that Trump has the emotional attitude towards Hillary expressed by crook.

(36) I totally respect Hillary, but Trump thinks that that crook should not be president.

While this fits well with the approach to inferring projection and causal and identity inferences sketched in the next section, one cannot do without accommodation in downwards entailing contexts such as the conditions of implications or the scope of negations. For these contexts, there are no extra beliefs of the speaker that would be equivalent to accommodations in the context.

What suffices in most cases is to assume a last resort possibility (a proper repair) where triggers are analysed as the conjunction of their presupposition and their semantic contribution.

A problem is however that this does not give a version of the quotation principle which says that if $S_{1}$ says So-and-so then this can be attacked by $S_{2}$ saying (37ab) where the attacked content is precisely the full conversational contribution of So-and-so.

a. It is not the case that So-and-so.

b. If So-and-so, I'll eat my hat.

The right solution seems to involve a number of strategies of which some are preferred over others.

\section{Inferring causation and identity}

Why do language interpreters make causal and identity inferences? And how? And when? As the question also applies to other forms of perception, it is more a question for artificial intelligence and cognitive psychology than for linguistics. The following is a sketch based on a stochastic and holistic version of the DRS generation algorithm.

There are many ways in which one cannot derive causal and identity inferences. First of all, such inferences add information and so it is not possible to obtain them by local probability maximation: adding information, the local probability goes down. Second, they do not follow from any of Grice's maxims, which squares well 
with the fact that they are also important in interpreting visual information: the Gricean maxims are all related to communication.

Third, it is not the case that propositions are accepted as true merely because they reach a probability threshold on their own. They only do if one has to make a choice. Typically we do not automatically infer that John is a diver if he has a titanium mouthpiece, even if that is - as we saw - very strong stochastic evidence that he is. It is especially important - as has been demonstrated in detail in the area of recognition of discourse structure - that such decisions involve different complete interpretations (Kehler (2002) makes this point in detail). It is always a question of comparing full explanations. Often identity inferences by themselves are not particularly probable. How probable is it that somebody has only skis as their means of transport as in (38)? This low probability must be compensated by the high probability that the speaker wants to indicate the consequences of the unfortunate event reported in the first sentence.

(38) Alena broke her skis. She lost her only means of transport.

Fourth, if one only looks at the content, the following situation obtains. The probability of an event $E$ can be equated with the probability of some event $D$ causing $E$. In some context $C$ therefore for any event $F$ given in $C$, the probability that $F$ causes $E$ is always smaller or equal than the probability of $E$ in the context.

The only way of breaking out of this quandary is to assume that causal inferences are due to a choice. Interpreters of visual information or verbally presented information on encountering a new event should make up their mind whether $E$ is caused by some given $F$ or whether it has an — as yet - hidden cause. This choice makes a lot of sense for visual information where information about its cause normally comes together with information about the event itself. One can assume that having to choose for verbally presented information is just the same system working in a situation where the speaker is in control and can choose not to present the cause with the event itself.

The same holds for identities. It is fundamental for visual information that one can identify the objects seen in two different perceptions, i.e. for a new perception of an object, decide whether it is a new perception of a given object or the perception of an as yet unknown object. Only in the latter case, a new discourse referent needs to be introduced. Again, one can assume that the same system deals with this question in verbal information, taking into account the specific linguistic means to force identities where intended (pronouns, descriptions, ellipsis and deaccentuation) and specific means to prevent identification (indefinite markers, other-marking, additive markers and contrastive intonation). The task with any new access to a discourse referent is to decide whether it was a new access to a given discourse referent or a new access to a new discourse referent. 
That means the interpretation system has to consider for each discourse referent occurring in the interpretation the possibility that it is identical or partly identical with each given discourse referent and the possibility that it is distinct from all of those.

For the causal inferences, it is possible to ride piggy-back on this identity check by introducing with each event its cause as a potentially new discourse referent. The cause will then be compared for identity with each discourse referent in the context.

Since the system also needs to make up its mind on the presupposition triggers, the same machinery applies here: if the presupposition is an event report (or a set of those), they get causes and hypothesized identities with given discourse referents. The hypothesis that the main referent is new comes with a disjunctive range of extra assumptions: a speaker belief in presupposition $P$ and possibly other assumptions involving $P$ that have the effect of local accommodations. If all these hypotheses fail, the last resort strategy of a assuming that trigger $T$ of presupposition $P$ exceptionally means $P \wedge T$.

The full interpretation must explain all the formal features (the words, the morphology, the constructions, the intonation) of the utterance and assign a speaker intention: a version of her state of mind that makes her production of the utterance plausible. This normally comes with a hypothesis about the question or questions the speaker tries to answer or the goal she tries to achieve.

This gives a potentially large but finite range of allowed hypotheses and the name of the game is to make a choice between them.

The best hypothesis is the one that is most probable in the context

Assume a context is given in the form $\langle\sigma, \pi\rangle$ where $\sigma$ is a set of worlds taken from a finite set $W$ representing the facts known in the context and $\pi$ is a set of probability distributions over the worlds in $W$ representing the causal dependencies. $\pi$ defines a partial pre-order over the sentences that are not decided by $\sigma$, as defined in (39).

$$
\begin{aligned}
& \text { a. } \left.p(\varphi)=\Sigma_{\{w \in \sigma: w \models \varphi}\right\} p(w) \\
& \text { b. }\langle\sigma, \pi>\models \varphi>\psi \text { iff } \forall p \in \pi p(\varphi)>p(\psi)
\end{aligned}
$$

It can now be defined what is an interpretation of an utterance $U$ with respect to $\langle\sigma, \pi\rangle$. It is a formula $\varphi$ without free variables such that produce $(\varphi, U)$ holds with respect to $\langle\sigma, \pi\rangle$ and for all $\psi$ other than $\varphi$ such that produce $(\psi, U)$ holds with respect to $\langle\sigma, \pi\rangle,\langle\sigma, \pi\rangle \models \varphi>\psi$.

It is not guaranteed that for any $U$ and $\langle\sigma, \pi\rangle$ there is such an interpretation. The speaker must do her part in the production of $U$ to make sure that the hearer will select the intended interpretation on the basis of common ground stochastic tendencies. 
$\varphi$ is then the conversational contribution of $U$ and should be an update for $\langle\sigma, \pi\rangle$, changing both the facts and the probabilities assumed. ${ }^{7} \varphi$ will contain causal inferences, identity inferences and preferences if these are preferred hypotheses.

The predicate produce $(\varphi, U)$ formalises a generation algorithm for the relevant language and should be seen as a set of causal generalisations. ${ }^{8}$

\section{Conclusion}

I argued that the empirical generalisation underlying the satisfaction theory, the LSE is not the correct generalisation and should be replaced by the CIG. The most important argument is formed by the cases, already noted by Karttunen where world knowledge needs to be invoked such as Mormon holy underwear, French snail eating and divers having wetsuits. Since these are generalisations with exceptions, the LSE is unable to predict the absence of projection in these cases. In the CIG, these are the basic cases and the cases that the LSE explains are cases where the inferences are trivial. It can be answered that we need a plausible reasoning extension of the LSE. In the face of the many obstacles for such an extension noted above, the CIG seems the best candidate for being just that extension.

The arguments of Van der Sandt against Karttunen in Van der Sandt (1988) and taken up again in Van der Sandt (1992) to the effect that the LSE predicts too much blocking are dealt with directly by the CIG: the CIG just gives the weaker version of LSE that is needed and one which is stronger than formal identity. The fact that the version of the LSE incorporated in Van der Sandt (1992) based on formal identity is too weak is already admitted in that paper: one needs further identifications based on conceptual knowledge. The CIG just gives what is needed without any further stipulations.

The most spectacular extra coverage of the extended CIG are the McCawley's LBJ-examples that have been outside the scope of any account of presupposition since the days of the Vietnam war in which they arose.

Causal and identity inferences reduce many cases of presupposition blocking to Trapping. The LSE-based accounts need dedicated presupposition-specific mechanisms to stop blocking. Those are unnecessary under the CIG.

It can be countered that causal and identity inferences are a high price for the simpler account of presupposition projection and that is true. It is however not possible to do NL interpretation without causal and identity inferences and all the extra complexity they bring is independently needed for effects that have no relation

7 The update system is part of forthcoming work and has many other applications.

8 Chapter 2 of Zeevat (2014) gives an account of the structure of produce $(x, y)$ and relates it to NL syntax and typology. 
to presupposition at all. It is also this aspect of the CIG from which the essential unity of presupposition with other kinds of projection can be seen.

Causal and identity inferences are an area to which NL semantics needs to devote more attention, if it is to retain its relevance for computational NLI and cognitive psychology.

\section{References}

Asher, Nicholas \& Alex Lascarides. 2003. Logics of Conversation: Studies in Natural Language Processing. Studies in Natural Language Processing. Cambridge University Press.

Beaver, David. 2010. Have you noticed that your belly button lint colour is related to the colour of your clothing? In Rainer Bauerle, Uwe Reyle \& Thomas Zimmermann (eds.), Presuppositions and Discourse: Essays Offered to Hans Kamp, Brill. doi:10.1163/9789004253162_004.

Beaver, David \& Henk Zeevat. 2007. Accommodation. In Gillian Ramchand \& Charles Reiss (eds.), Oxford Handbook of Linguistic Interfaces, 503-540. Oxford University Press. doi:10.1093/oxfordhb/9780199247455.013.0017.

Garcia-Odon, Amaia. 2016. Presupposition projection and conditionalization. Topoi 35(1). 145-156. doi:10.1007/s11245-014-9279-y.

Gazdar, Gerald. 1979. Pragmatics: Implicature, Presupposition and Logical Form. New York: Academic Press.

Heim, Irene. 1983. On the projection problem for presuppositions. In Michael Barlow, Daniel Flickinger \& Michael Westcoat (eds.), Second Annual West Coast Conference on Formal Linguistics, 114-126. Stanford University.

Hobbs, Jerry R. 1985. On the coherence and structure of discourse. Tech. Rep. CSLI85-37 Center for the Study of Language and Information, Stanford University.

Jasinskaja, Ekaterina. 2007. Pragmatics and prosody of implicit discourse relations: The case of restatement: University of Tübingen $\mathrm{PhD}$ dissertation.

Kamp, Hans \& Uwe Reyle. 1993. From Discourse to Logic. Kluwer. doi:10.1007/978-94-011-2066-1.

Karttunen, Lauri. 1973. Presuppositions of compound sentences. Linguistic Inquiry 4(2). 167-193.

Karttunen, Lauri. 1974. Presupposition and linguistic context. Theoretical Linguistics 1(1-3). doi:10.1515/thli.1974.1.1-3.181.

Karttunen, Lauri \& Stanley Peters. 1979. Conventional implicatures in Montague Grammar. In Choon-Kyu Oh \& David Dineen (eds.), Syntax and Semantics 11: Presupposition, 1-56. New York: Academic Press.

Kehler, Andrew. 2002. Coherence, Reference, and the Theory of Grammar. CSLI Publications. 
Local Satisfaction explained away

Kehler, Andrew, Laura Kertz, Hannah Rohde \& Jeffrey L. Elman. 2008. Coherence and coreference revisited. Journal of Semantics 25(1). 1-44. doi:10.1093/jos/ffm018.

Kripke, Saul. 2009. Presupposition and anaphora: Remarks on the formulation of the projection problem. Linguistic Inquiry 40(3). 367-386. doi:10.1162/ling.2009.40.3.367.

Langendoen, D. Terence \& Harris Savin. 1971. The projection problem for presuppositions. In Charles Fillmore \& D. Terence Langendoen (eds.), Studies in Linguistic Semantics, 373-388. New York: Holt, Reinhardt and Winston.

Potts, Christopher. 2005. The Logic of Conventional Implicatures. Oxford University Press. doi:10.1515/9783110253382.2516.

Rothschild, Daniel. 2011. Explaining presupposition projection with dynamic semantics. Semantics and Pragmatics 4(3). 1-43. doi:10.3765/sp.4.3.

Van der Sandt, Rob. 1988. Context and Presupposition. London: Croom Helm.

Van der Sandt, Rob. 1992. Presupposition projection as anaphora resolution. Journal of Semantics 9. 333-377. doi:10.1093/jos/9.4.333.

Schlenker, Philippe. 2007. Transparency: An incremental theory of presupposition projection. In Presupposition and Implicature in Compositional Semantics, 214-242. Springer Nature. doi:10.1057/9780230210752_8.

Simons, Mandy, Judith Tonhauser, David Beaver \& Craige Roberts. 2011. What projects and why. In D. Lutz \& N. Li (eds.), Semantics and Linguistic Theory XX, Cornell: CLC Publications. doi:10.3765/salt.v0i20.2584.

Soames, Scott. 1982. How presuppositions are inherited: A solution to the projection problem. Linguistic Inquiry 13. 483-545.

Zeevat, Henk. 1992. Presupposition and accommodation in update semantics. Journal of Semantics 9(4). 379-412. doi:10.1093/jos/9.4.379.

Zeevat, Henk. 2002. Explaining presupposition triggers. In Kees van Deemter \& Rodger Kibble (eds.), Information Sharing: Reference and Presupposition in Language Generation and Interpretation, CSLI.

Zeevat, Henk. 2014. Language Production and Interpretation. Linguistics Meets Cognition. Jacob Brill.

Henk Zeevat

SFB991

Heinrich-Heine-Universität Düesseldorf

henk.zeevat@uva.nl 